\title{
The influence of tension mean stress to the crack initiation process at the very high cycle fatigue regime for a high strength steel
}

\author{
Thomas Rohm ${ }^{1,2}$, Karl-Heinz Lang ${ }^{1, *}$, and Marcus Korn ${ }^{1}$ \\ ${ }^{1}$ Karlsruhe Institute of Technology (KIT) Institute for Applied Materials (IAM), Engelbert-Arnold-Str. 4, 76131 Karlsruhe, Germany \\ ${ }^{2}$ Karlsruhe University of Applied Sciences, Moltkestr. 30, 76133 Karlsruhe, Germany
}

\begin{abstract}
Strain controlled tests were performed on a high strength steel to investigate the influence of tension mean stress to the crack initiation process at the very high cycle fatigue regime. It is supposed that the variation of stress the state in the vicinity of a non-metallic inclusion results in a change to the energy transferred to the surrounding metallic matrix. To prove this, incremental step tests on cylindrical specimen were realized in which the tension mean stress was stepwise increased. It could be verified that the hysteresis area, which is a criterion of the work transferred to the specimen, decreases with increasing tension mean stress. The test results provide a reasonable physical model to estimate the influence of tension mean stress to the crack initiation process in the very high cycle fatigue regime.
\end{abstract}

\section{INTRODUCTION}

Some investigators like $[1,2,3]$ have shown, that compressive residual stresses at the surface of specimens due to local plastic deformation or local plastic deformation and reduction of surface roughness, increases the lifetime also in the very high cycle regime. From these results it is known, that the compressive mean stress inhibits the crack initiation at or close to the surface, until the influence is compensated by crack initiation in the volume of the specimen. A specific influence resulted from the rate of tension residual stress at the specimen volume was not mentioned.

Considering the residual stress using the formula of Murakami [4] for the stress intensity factor (SIF) $K_{\max }$, a particular effect can be assigned. To calculate $K_{\max }$ the following equitation (1) is used, with $\Delta \sigma / 2=$ nominal stress and $\sigma^{R S}=$ residual stress in MPa, $S_{x z}=$ inclusion area in $\mathrm{m}^{2}$ and $f=0.5$ for subsurface defects or $f=0.65$ for surface defects.

$$
K_{\max }=f \cdot\left(\Delta \sigma / 2+\sigma^{R S}\right) \cdot \sqrt{\pi \sqrt{S_{x z}}}
$$

Tension residual stresses increase the stress intensity factor at an inclusion. Therefore, the size of the optically dark area (ODA) [4] which is necessary to achieve the long crack growth threshold value is smaller as without residual stresses or even larger if compressive residual stresses are present as illustrated in fig. 1. But a different size of the ODA is related to a different lifetime of a specimen failing in the VHCF-regime $[5,6]$. Accordingly, a modification of the residual stresses in the volume of a specimen will also cause a change in the lifetime. Higher tension residual stresses in the volume of a specimen due to higher or deeper reaching compressive residual stresses at the surface should result in a shorter lifetime at the very high cycle fatigue regime because in this lifetime regime crack initiation typically takes place in the volume of the specimen where tensile residual stresses are effective.

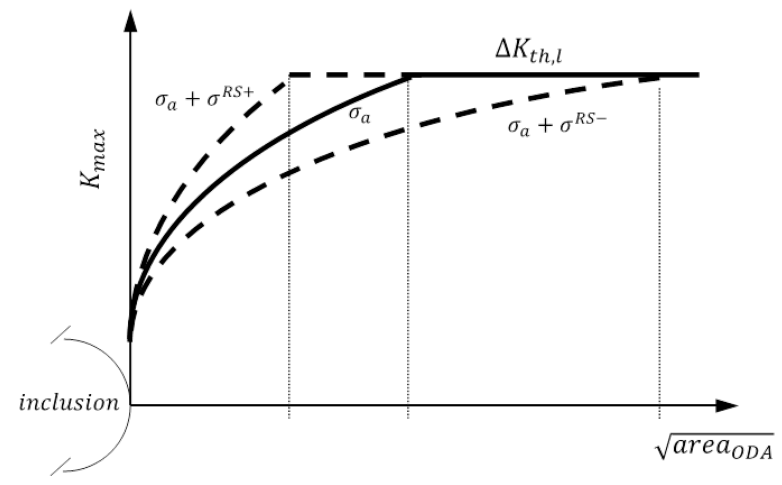

Fig. 1. Influence of residual stresses to the dimension of ODA until the long crack growth threshold is reached.

In [7] is reported, that a lager compressive residual stress fields resulting from shot peening lead to longer lifetime in the range between $N=10^{7}$ to $N=10^{9}$ cycles compared to slight compressive residual stresses produced by mechanical polishing. Similar results are reported in [8] where macro shot peened specimens exhibited longer lifetimes compared to micro peened specimens. Fig. 2 shows fatigue test results in the VHCF-regime [8], that if crack initiation at an inclusion in the volume with a formation of an ODA is present, higher residual tensile stresses in the volume as a result of higher compressive residual stress near the specimen surface dose not reduce the lifetime. These results are in contrast to the usual considerations of the impact of residual stresses to the lifetime behavior. This is due to the fact that residual stresses have different effects in the different phases of fatigue. Tensile residual stresses accelerate the growth of macroscopic cracks and thus reduce the lifetime. But in the VHCF-regime with

\footnotetext{
${ }^{*}$ Corresponding author: karl-heinz.lang@,kit.edu
} 
formation of an ODA at an inclusion the proportion of long crack growth of the total fatigue life is marginal [9]. Therefore, the influence of residual stresses on the crack initiation process (including the formation of ODAs) and on short fatigue crack growth must be considered. The impact of residual stresses in these phases can differ greatly from the effect on the long crack growth phase.

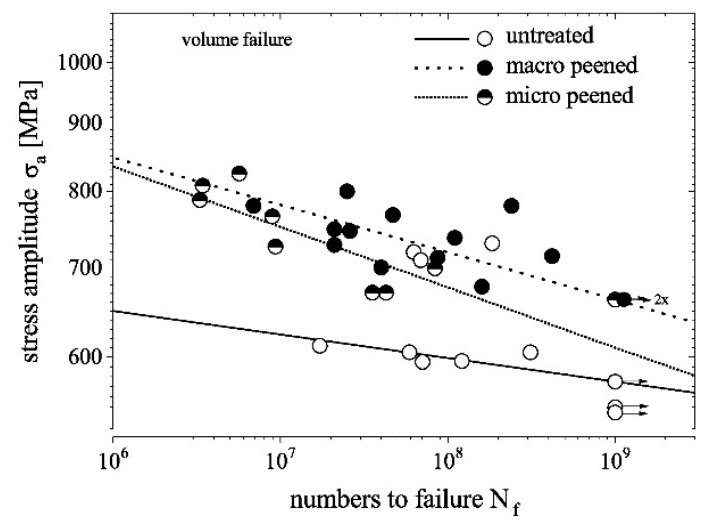

Fig. 2. Endurance test with the steel AISI 4140, $R_{m}=2145 \mathrm{MPa}$ under tension compression load at $R=-1$. Surface condition: untreated, macro peened $(0.29 \mathrm{mmA})$ and micro peened (glas beeds) [8].

The purpose of the present study is a selective evaluation of the impact of tension residual stresses on the crack initiation process. It is supposed that tension residual stresses which are effective in the surrounding of a non- metallic inclusion, where cracks are initiated in the VHCF-regime for high strength steels [10], leads to a reduction of the energy transferred to the specimen by the cyclic loading. Most of this work will dissipate by heat, but a small amount will be stored in the material, cause microstructural changes and thereby, induce fatigue damage in the material [11]. Because the crack initiation process in the VHCF-regime may take up to $99 \%$ of the lifetime [9], the influence of residual stresses to this process dominates the lifetime behavior. Therefore, the working hypothesis is that tension residual stresses may extend the lifetime in the VHCFregime by reducing the energy transferred to the matrix surrounding a non-metallic inclusion.

For the following tests specific assumptions are necessary: For the used steel in this study $\mathrm{AlCaO}$ oxides which have a globular shape are typically responsible for crack initiation [12]. It is assumed, that the shape of these inclusions is ideal spherical and that they are detached from the metallic matrix [13]. Therefore, the loading condition in the surrounding matrix is comparable to a notch when tensile stresses are acting. In the case of compressive stresses the existing inclusion can transfer loading and only minor effects of stress concentration appear as long as the different elastic properties of the matrix and the inclusion are neglected. For further information see [14]. Thus the external load ration at the specimen of $R=-1$ results in a higher load ration of about $R=-0.5$ at the inclusion if an elastic stress concentration of $K_{t}=2.045$ for spherical cavity and with a Poisson's ratio $v=0.3$ are assumed [15]. The influence of different residual stresses will be emulated by the variation of the mean load. In the case of overelastic deformation the loading conditions in the notch root is determined by the elastic behavior of the nonplasticizing regions. Therefore, total strain control is required to emulate the loading conditions in the vicinity of a non-metallic inclusion on a smooth specimen.

\section{EXPERIMENTAL PROCEDURES}

The test material used in this study is the low alloyed steel 42CrMo4 (AISI 4140) in quenched and tempered condition. The chemical composition (mass percentage) of this steel is: $0.422 \mathrm{C}, 1.062 \mathrm{Cr}, 0.851 \mathrm{Mn}, 0.162 \mathrm{Mo}$, $0.299 \mathrm{Si}, 0.021 \mathrm{~S}, 0.016 \mathrm{P}$ and balance Fe. The specimens were machined from a $15 \mathrm{~mm}$ rolled round bar to cylindrical specimens with a gauge section length of $10 \mathrm{~mm}$ and a diameter of $4 \mathrm{~mm}$. The surface roughness was $R_{z}=2.79 \mu \mathrm{m} \pm 0.172 \mu \mathrm{m}$ (average and standard deviation). After machining the specimens were austenitised at $850^{\circ} \mathrm{C}$ in vacuum for $20 \mathrm{~min}$, oil quenched and tempered at $180^{\circ} \mathrm{C}$ for $180 \mathrm{~min}$. The hardness was $611 \pm 11 \mathrm{HV} 0.1$ (average and standard deviation), the ultimate tensile strength was $2145 \mathrm{MPa}$ and the yield strength was $1730 \mathrm{MPa}$. To get an effective determination of the cyclic stress-strain-curve of the material in a low amount of testing time, the incremental step test is a common used investigation [16]. Because the influence of tension stress is focused, which is considered to be equivalent to mean stress, the test procedure is modified. Constant strain amplitude with variable mean strain will be executed and sequentially repeated to stabilize the stress-strain behaviour of the material.

The constant strain amplitude was $\varepsilon_{a}=0.6 \%$, the maximum total mean strain was $\varepsilon_{m, t}=0.5 \%$ and for the test a frequency of $f=0.5 \mathrm{~Hz}$ was applied. To avoid an early failure of the specimen, the maximum total strain was chosen at a level, were a sufficient number of cycles are possible [17]. Theses investigations showed, that the material tends to cyclic softening until the final fracture, however it is not very distinct. The main softening process is the resolution of coherent $\varepsilon$-carbide precipitates due to cyclic loading, therefore an increased movement of slip of dislocations is possible. But this softening effect is limited, as result of [17] show. It is expected that the stabilization of the cyclic softening will develop after 10 or more sequence cycles. While the strain amplitude was constant, the total mean strain was stepwise exceeded. This variation represents the mean strain, which exists because of additional stress, likewise residual stress. At each mean strain level fife loops were performed to get closed hysteresis loops for the data evaluation. The area of the stress-strain hysteresis loop is equivalent to the transferred energy. Fig. 3 explores one sequence schematically and the test set up at the specimen for stain measurement. 

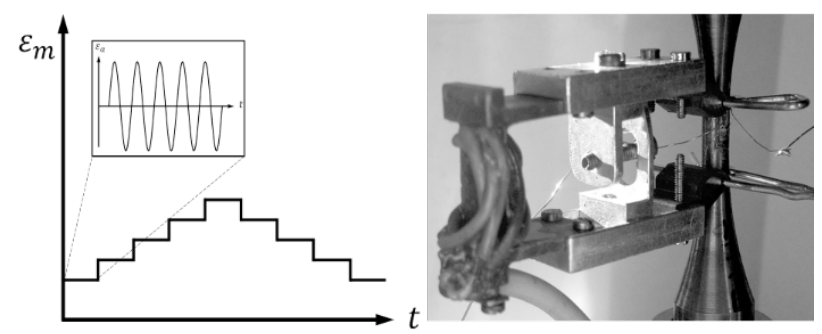

Fig. 3. One schematically test sequence and stain measurement setup at the specimen.

The stabilization was monitored comparing the maximum values of stress-strain-curves at the sequences. While the softening at the beginning was significant, after the 11th repetition the following sequences showed minor softening, so these sequences were evaluated. The stress and strain data were stored on a computer and automatically evaluated.

\section{EXPERIMENTAL RESULTS AND DISCUSSION}

Evaluating the data of the 13th sequence the plastic strain amplitude of the hysteresis loops is linear increasing with higher total mean strain, as fig. 4 illustrates. At the same time stress amplitude is decreasing, but with higher mean strain the reduction is clearly larger.

At higher mean stress the maximum stress exceeds the cyclic yield strength and hardening mechanisms occur. With increasing maximum total strain, a higher number of slip dislocations at different levels are activated at the polycrystalline material. The interaction of the dislocations and the pile up at micro structural barriers, like coherent $\varepsilon$-carbide precipitations, create a less increase of hardening. An extension of external stress enables dislocations to circumvent the carbides and cross slip enables their extinction.

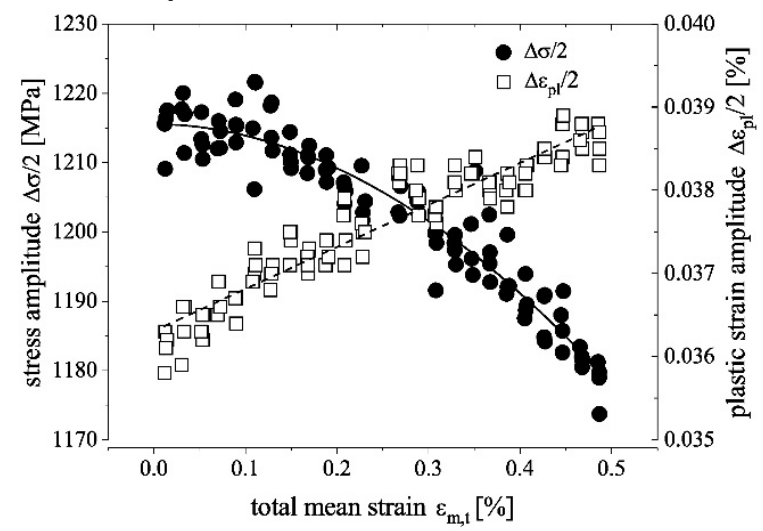

Fig. 4. Variation of maximum stress $\Delta \sigma / 2$ and plastic strain amplitude $\Delta \varepsilon / 2$ at different total mean strain.

As a result of cyclic stress-strain behavior of the material, the hysteresis loop area is increasing as the plastic strain amplitude increases, as fig. 5 illustrates. There the hysteresis area represents the specific work $W$ which is transferred at the closed loop. At a total mean strain of $\varepsilon_{m, t}=0.3 \%$ and a strain ratio of $R=-0.32$ the hysteresis area is unexpectedly decreasing. Macroscopic voids at the material surface could not be found and the analysis of the specimen stiffness provides not a reasonable explanation for this phenomenon.

It is supposed that a specific material softening is necessary to raise this effect. While the plastic strain is increasing constantly, an over proportional decrease of the stress amplitude with higher mean strain, results in a smaller hysteresis loop area.

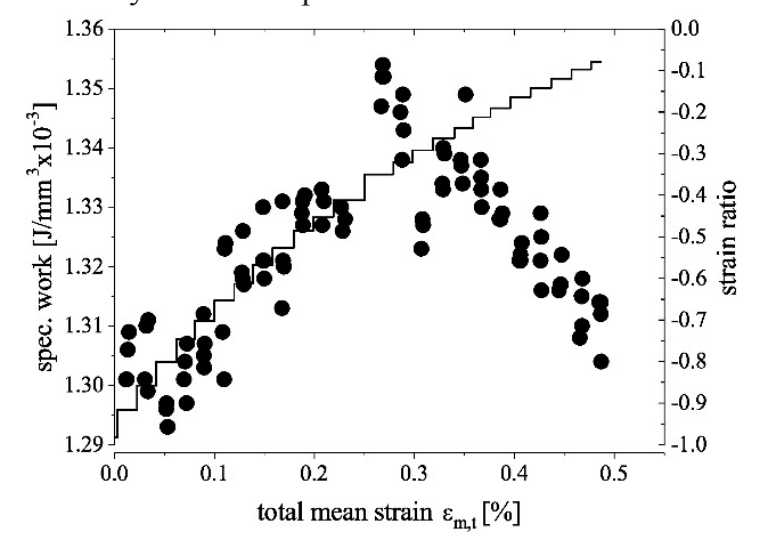

Fig. 5. Specific work $W$ transferred of the hysteresis loop and strain ratio $R$ depending on increasing total mean strain $\varepsilon_{m, t}$.

In contrary to the influence of tensile mean stress, where the growth of macroscopic cracks is accelerated and live time is decreased [18], this findings are evident suggests that the reduced hysteresis area could result in an increase of life time in the VHCF-regime. So less energy is transferred in the metallic matrix at the vicinity of the non-metallic inclusion per load cycle and a higher number of cycles is necessary to achieve the same amount of energy to initiate the crack growth.

A quantitative relationship between the minor specific work at the hysteresis loop, caused by increased mean strain and extended lifetime, derived from this results, cannot be provided at the moment. Further tests are necessary to investigated how much energy is dissipated within heat and how much is stored at the material for the crack initiation process in the VHCFregime. With this information a more detailed correlation between residual tension stress and lifetime for at nonmetallic inclusions initiated cracks with ODA-formation, could be realized.

\section{SUMMARY}

Within the scope of this research, it was shown that there is a sufficient influence of the tension residual stress which could result in a reduced transferred energy in the surrounding material of a spherical non-metallic inclusion. A significant incident could be identified that with increasing mean strain and constant strain amplitude less energy is transferred in the material. While the crack initiation process with ODA-formation is dominant in the VHCF-regime, the minor specific work per cycle can prolong the life time. Fig. 6 illustrates the influence of compressive residual stress near the surface and tension residual stress in the volume of the specimen, to the fatigue behavior in the VHCFregime. The compressive stresses inhibit the crack initiation at or close to the surface (S) so a failure from 
the volume (VO) of the specimen can occur. But with higher compression residual stresses and therefor higher tension stresses at the volume, there will be an additional prolongation of lifetime, when ODA-formation in the VHCF-regime is present.

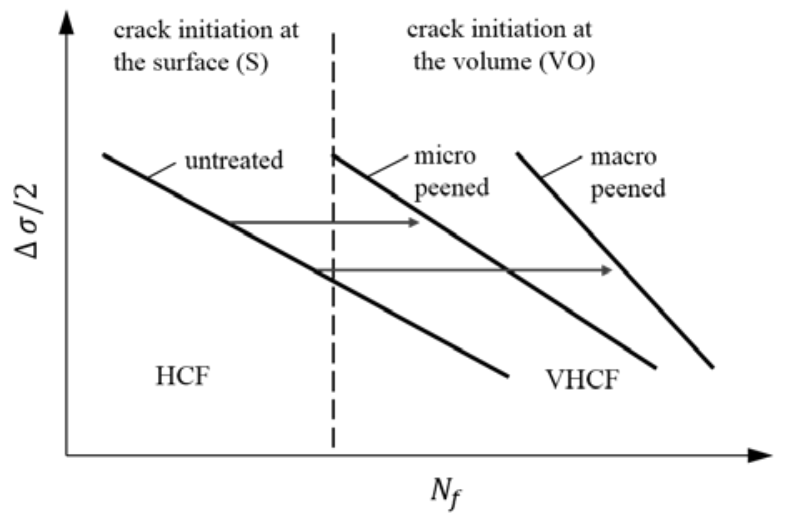

Fig. 6. Influence to the lifetime in the VHCF-regime due to low amount of residual stress by a procedure of micro peening and high amount of residual stress by macro peening.

This study provides a physically proven basis to assess the influence of mean stress in a new way for the VHCF-regime. The material behaviour is not fully investigated. The formation of the dislocations would be a helpful investigation to understand this phenomenon in detail. But this material configuration has already a very high dislocation density so any change is complex to detect and the evaluation would have a high uncertainty. Further investigations with a model material, like copper or pure iron are necessary. In an ideal material behaviour the softening would be constant with increasing mean strain so this influence could be eliminated. Also tests with minor changes of the stress ratio would be interesting, because results for higher stress ratio already exists [19].

\section{ACKNOWLEDGEMENTS}

The authors would like to thank the German Research Foundation (DFG) for the financial support at the SPP1466.

\section{REFERENCES}

1. C. R. Sohar, A. Betzwar-Kotas, C. Gierl, B. Weiss, H. Danninger, Materialwissenschaft und Werkstofftechnik 39 248-257 (2008)

2. I.-S. Cho, A. Amanov, D.-W. Kim, H.-J. Park, B.H. Kim, C.-S. Lee, Y.-S. Pyoun, I.-G. Park, VHCF5 341-346 (2011)

3. Y. Shimatani, K. Shiozawa, T. Nakada, T. Yoshimoto, Procedia Engineering 2873 - 882 (2010)

4. Y. Murakami, Metal Fatigue, (2002)

5. Y. Murakami, T. Nomoto, T. Ueda, Y. Murakami, Fatigue \& Fracture of Engineering Materials \& Structures 23 893-902 (2000)
6. K. Shiozawa, L. Lu, Fatigue \& Fracture of Engineering Materials \& Structures 25 813-822 (2002)

7. N. Shiraki, S. Abe, Y. Sugiyama, C. Braham, VHCF3, 601-608 ( 2004)

8. M. Korn, T. Rohm, K.-H. Lang, Springer Spektrum, (to be published)

9. Q. Wang, C. Bathias, N. Kawagoishi, Q. Chen, IJF 24 1269-1274 (2002)

10. Y. Murakami, S. Kodama, S. Konuma, IJF 11 291298 (1989)

11. M. Bever, D. Holt, A. Titchener, Progress in Materials Science 5-177 17 (1973)

12. K.-H. Lang, M. Korn, T. Rohm, Procedia Structural Integrity 2 1133-1142 (2016)

13. Y. Murakami, N. N. Yokoyama, J. Nagata, Fatigue \& Fracture of Engineering Materials \& Structures 25 735-746 (2002)

14. A. Stienon, A. Fazekas, J.-Y. Buffiere, Materials Science and Engineering: A 513-514 376-383 (2009)

15. S. Timoshenko, Theory of elasticity, Engineering societies monographs, (1951)

16. H.-J. Christ, Werkstoff-Forschung und Technik 9 (1991)

17. P. N. Thielen, M. E. Fine, R. A. Fournelle, Acta Metallurgica 24 1-10 (1976)

18. R. Stephens, A. Fatemi, R. Stephens, H. Fuchs, Metal Fatigue in Engineering, (2000)

19. Y. Furuya, T. Abe, Materials \& Design 32 11011107 (2011) 